BMJ

Open

Gastroenterology

\title{
Duodenal biopsies for the diagnosis of coeliac disease: are we adhering to current guidance?
}

\author{
Nilofer Husnoo, ${ }^{1}$ Wafaa Ahmed, ${ }^{2}$ Muhammad Hanif Shiwani ${ }^{3}$
}

To cite: Husnoo N, Ahmed W, Shiwani MH. Duodenal biopsies for the diagnosis of coeliac disease: are we adhering to current guidance?. BMJ Open Gastro 2017:4:e00140.

doi:10.1136/bmjgast-2017000140

Received 20 February 2017 Revised 20 March 2017 Accepted 21 March 2017

\section{${ }^{1}$ Urology Department, Sheffield Teaching Hospitals NHS Trust, Sheffield, UK ${ }^{2}$ Gastroenterology Department, Maidstone and Tunbridge Wells NHS Trust, Tunbridge Wells, UK ${ }^{3}$ General Surgery Department, Barnsley General Hospital NHS Foundation Trust, Barnsley, UK}

Correspondence to Nilofer Husnoo; niloferhusnoo@doctors.org. uk

\section{ABSTRACT \\ Background: The British Society of Gastroenterology guidelines recommend taking at least four duodenal biopsy specimens at the time of upper gastrointestinal (UGI) endoscopy if coeliac disease (CD) is suspected and it has been shown to increase the diagnostic yield of $\mathrm{CD}$. We assessed the compliance to these guidelines within our institution. We then applied measures to improve our compliance rate and assessed the resulting impact on our diagnostic rate of $C D$.}

Methods: We performed a retrospective audit of electronic records for all patients, with no prior diagnosis of $C D$, who underwent UGI endoscopy with duodenal biopsies between August 2014 and May 2015. We implemented measures to raise awareness among endoscopy users at our institution and carried out a reaudit between February and May 2016.

Results: 924 patients were found to be eligible in the first part of the study and 278 in the second part. The proportion of patients who had $\geq 4$ biopsy specimens submitted increased from $21.9 \%$ to $60.8 \%(p<0.001)$. The diagnostic rate of $C D$ increased from $3.5 \%$ in the audit group to $7.6 \%$ in the reaudit group $(p=0.004)$. A positive serology result and suspected $C D$ as an indication for biopsy were found to be independent predictors of the likelihood of complying with guidelines.

Conclusions: Our study suggests that taking $<4$ duodenal biopsy specimens to assess for the presence of $C D$ may lead to the diagnosis of $C D$ being missed. Simple measures can improve the local compliance rate to current guidelines.

\section{INTRODUCTION}

Coeliac disease (CD) is a common condition with a prevalence of at least $1 \%$ in the $\mathrm{UK}^{1}$ $\mathrm{CD}$ can present with a range of symptoms including vague and non-specific abdominal symptoms similar to irritable bowel syndrome. $^{2}$ With time, the proportion of patients presenting with atypical symptoms has also increased. ${ }^{3}$ Thus, it is important to ensure that when $\mathrm{CD}$ is considered as a potential diagnosis, patients are adequately investigated to avoid missing the diagnosis. The lifetime risk of malignancy, especially

\section{Summary box}

- Mucosal changes can be patchy in coeliac disease (CD).

- The British Society of Gastroenterology (BSG) recommends taking at least four biopsy specimens if $C D$ is suspected at the time of endoscopy.

- Previous studies have shown that this increases the diagnostic rate of $C D$.

- Despite existence of clear BSG guidance, compliance to this is quite poor in our unit, which may reflect a more widespread problem.

- This may be due to a lack of awareness of guidelines, or other barriers.

- Simple interventional measures may help to improve compliance and potentially improve the diagnostic rate of $\mathrm{CD}$.

- Our results should prompt other units to audit their practice and implement measures to improve adherence to current recommendations, and thus the care of patients with potential $C D$.

intestinal lymphoma and other gastrointestinal cancers, in fact, slightly increased in $\mathrm{CD}^{4}$ with studies quoting an incidence of lymphoma of $8 \% .^{5}$

Endoscopy remains the mainstay of adult $\mathrm{CD}$ diagnosis. As histological changes in CD can be patchy, several biopsies should be taken from the bulb and more distal duodenum at the time of endoscopy. Updated guidelines from the British Society of Gastroenterology (BSG) on the management of adult CD recommend taking at least four duodenal biopsies, including one from the bulb, if CD is suspected. ${ }^{6}$ This is based on a large study involving 132352 patients without known $\mathrm{CD}$, which showed that taking $\geq 4$ duodenal biopsy samples resulted in a doubling of the diagnostic rate of $\mathrm{CD}$ compared with when $<4$ samples were taken. ${ }^{7}$ This is further supported by evidence from a retrospective study involving patients with known $\mathrm{CD}$, which showed that the sensitivity for a diagnosis of $\mathrm{CD}$ increased with incremental 
biopsies, and the diagnosis could be confirmed in $100 \%$ of patients when four biopsy specimens were taken. ${ }^{8}$

We carried out a study to assess our compliance to the BSG guidelines in patients without known CD. Following implementation of measures to improve compliance, we also aimed to determine the influence of better compliance to guidelines on our local diagnostic rate of $\mathrm{CD}$.

\section{METHODS}

A list of all patients who had endoscopic duodenal biopsies submitted for histopathological analysis to the Barnsley General Hospital NHS Foundation Trust (UK) pathology laboratory over a 10-month period from August 2014 to May 2015 was retrieved from the laboratory database using coding. For each set of specimens submitted, the date of specimen collection, the clinician performing the biopsy, and patient age and sex were also available. Using the Integrated Clinical Environment (ICE-the online system for pathology reporting), we obtained information on the main indication for biopsy, the number of biopsy samples for each specimen, histopathology report and results of IgA anti-tTG antibody tests if performed (as this is the most widely available serology test for CD screening locally especially in primary care).

We presented the results of this first part of our study at the local Endoscopy User Group meeting in November 2015. This meeting is attended by endoscopists in the trust who have regular endoscopy lists and therefore perform the majority of endoscopies in the unit, as well as endoscopy nurses. While most of these clinicians were present, a number did not attend. We therefore also put up posters summarising the results of our findings in all clinical areas within the endoscopy unit to raise awareness of the importance of complying with guidelines among endoscopists and to encourage and remind them to take the appropriate number of biopsies. We then collected data using the same methods described above for duodenal biopsies submitted for histopathological analysis over a 3-month period from February 2016 to May 2016.

As clinical records were not accessed, for the purpose of this study CD was defined as:

I. either histological Marsh grades IIIa, IIIb or IIIc (as defined by the Modified Marsh classification);

II. Marsh grades I or II or a report of increased intraepithelial lymphocytosis in conjunction with a positive serology result (anti-tTG antibody positive).

These definitions are based on the algorithm for the diagnosis of CD laid out in the BSG guidelines. ${ }^{6}$ In our institution, an IgA-tTG antibody level of $10 \mathrm{U} / \mathrm{mL}$ is regarded as definitely positive. Values of exactly 10U/ $\mathrm{mL}$ or just under $10 \mathrm{U} / \mathrm{mL}$ are reported as either weakly positive or equivocal-we have regarded these as negative results for the purpose of our study.

The study was registered with and approved by the trust audit department, which did not deem local research and development approval necessary due to the study being purely an audit.

\section{Exclusion criteria}

Patients with known CD, having duodenal biopsies for follow-up, were excluded. We also excluded records where the number of biopsy specimens was not specified (described as 'several' or 'multiple'). For patients having more than one endoscopy examination with duodenal biopsies during one study period, we only considered the first endoscopy. Duodenal bulb specimens (when taken and specified in the histopathology report) were included in the total count of number of duodenal specimens submitted.

\section{Statistical analysis}

The $\chi^{2}$ test was used to compare categorical variables and the independent t-test to compare means of numerical variables between the audit and reaudit groups. We also used the $\chi^{2}$ test to assess the impact of compliance to BSG guidelines on the proportion of patients diagnosed with CD (as per criteria defined above). We used a generalised estimating equation (GEE) to identify factors predicting adherence to guidance, while adjusting for clustering by endoscopist. All $p$ values are 2-sided. The IBM SPSS (V.24) software was used for all statistical analyses.

\section{RESULTS}

A total of 973 endoscopies with duodenal biopsies were performed from August 2014 to May 2015. Of these, 924 endoscopies were included in the study after applying exclusion criteria. The respective figures for the reaudit from February 2016 to May 2016 were 290 and 278. Table 1 shows the characteristics of patients included in the study. There were no significant differences between groups in terms of patient demographics and indication for biopsy. These 1263 endoscopies with duodenal biopsies performed across the two study periods (before applying exclusion criteria) represent $27.5 \%$ of the total number of upper gastrointestinal endoscopies performed in our institution.

In the first part of the study, $\geq 4$ biopsy specimens were submitted for histopathological analysis in $21.9 \%$ of cases $(n=219)$. This increased to $60.8 \%(n=169)$ in the reaudit following implementation of interventional measures $(\mathrm{p}<0.001)$. Even when 'suspected coeliac disease/ malabsorption' was the indication for biopsy, 39.2\% $(n=47)$ of patients had $\geq 4$ biopsies taken in the first study period, but the corresponding figure was $60.0 \%$ $(\mathrm{n}=18)$ in the reaudit $(\mathrm{p}=0.039)$. The modal number of specimens submitted also changed from 3 to 4 (figure 1A,B).

Thirty-two patients were found to have $\mathrm{CD}$ in the first cohort and 21 in the second $(3.5 \%$ vs $7.6 \%, \mathrm{p}=0.004)$. In both groups of study, before and after intervention, the diagnostic yield of $\mathrm{CD}$ when $\geq 4$ biopsies were taken was 
Table 1 Characteristics of patients, without known coeliac disease, undergoing endoscopy and duodenal biopsies

\begin{tabular}{|c|c|c|c|c|c|}
\hline & \multicolumn{2}{|c|}{$\begin{array}{l}\text { Audit } \\
\text { (Aug 2014-May 2015) }\end{array}$} & \multicolumn{2}{|c|}{$\begin{array}{l}\text { Reaudit } \\
\text { (Feb 2016-May 2016) }\end{array}$} & \multirow[b]{2}{*}{ p Value } \\
\hline & Number & Per cent & Number & Per cent & \\
\hline Total number of endoscopies & 973 & & 290 & & - \\
\hline Number included in study & 924 & - & 278 & - & \\
\hline Sex & & & & & 0.705 \\
\hline Males & 374 & 40.5 & 109 & 39.2 & \\
\hline Females & 550 & 59.5 & 169 & 60.8 & \\
\hline \multicolumn{6}{|l|}{ Age (years) } \\
\hline Mean & 59.2 & & 59.7 & & 0.692 \\
\hline Range & $15.6-91.6$ & & $19.6-95.6$ & & \\
\hline Indication & & & & & 0.512 \\
\hline Anaemia & 469 & 50.8 & 155 & 55.8 & \\
\hline Suspected coeliac/malabsorption & 120 & 13.0 & 30 & 10.8 & \\
\hline Epigastric pain/dyspepsia & 108 & 11.7 & 30 & 10.8 & \\
\hline Weight loss & 97 & 10.5 & 32 & 11.5 & \\
\hline Diarrhoea & 81 & 8.8 & 16 & 5.8 & \\
\hline Reflux & 22 & 2.4 & 5 & 1.8 & \\
\hline Others & 27 & 2.9 & 10 & 3.6 & \\
\hline \multicolumn{6}{|l|}{ Serology test } \\
\hline Performed & 465 & 50.3 & 126 & 45.3 & 0.144 \\
\hline Positive & 37 & 4.0 & 18 & 6.5 & 0.084 \\
\hline
\end{tabular}

higher than when $<4$ biopsies were taken. The diagnostic rate of $\mathrm{CD}$ in the audit group was $2.0 \%$ (14 patients out of 705$)$ when $<4$ biopsies were taken and $8.2 \%(18$ out of 219 patients) when $>4$ biopsies were taken $(\mathrm{p}<0.001)$. The respective figures were $3.7 \%$ increasing to $10.1 \%$ with $\geq 4$ biopsies in the reaudit group $(p=0.049)$. The results from both parts of the study are summarised in table 2 .

A total of 42 different clinicians performed endoscopies with duodenal biopsies in the 1202 patients (audit and reaudit groups combined). Eighteen of these clinicians performed at least 10 endoscopies, with the remaining 24 performing fewer than 10 endoscopies over the study periods considered. Between these 18 clinicians, a total of 1133 endoscopies were performed (ie, $94 \%$ of the overall number of endoscopies performed). Their overall compliance with guidelines varied from $12 \%$ to $67 \%$ (SD $11.9 \%$ ).

We therefore identified factors predicting overall adherence to BSG guidelines (ie, submission of $\geq 4$ biopsy specimens), while adjusting for clustering by clinician, using GEE. The following parameters were assessed as predictors of taking $\geq 4$ biopsies: study group (audit vs reaudit), age, sex, indication for biopsy and serology test result (positive or negative).

The reaudit group was associated with a significantly increased odds of taking four or more biopsies compared with the audit group (OR 5.3; 95\% CI 3.8 to 7.5; $\mathrm{p}<0.001)$. Suspected CD as an indication for biopsy also had a higher odds of adhering to guidance (OR 2.45; $95 \%$ CI 1.01 to 6.17 ; $<0.05$ ), while the lack of a positive anti-tTG antibody result was associated with decreased odds of compliance (OR $0.39 ; 95 \%$ CI 0.21 to 0.73 ; $\mathrm{p}=0.003)$. The remaining factors considered did not reach statistical significance.

\section{DISCUSSION}

Only a quarter of upper gastrointestinal endoscopies performed in our institution result in duodenal biopsies, which reinforces the fact that, when taken, the main aim probably remains to diagnose or exclude CD. The results of our initial audit thus demonstrate poor adherence to established national recommendations in
Figure 1 Bar charts of number of duodenal biopsies submitted for histopathological analysis for patients having an endoscopy and duodenal biopsies between (A) August 2014 and May 2015 and (B) February 2016 and May 2016.
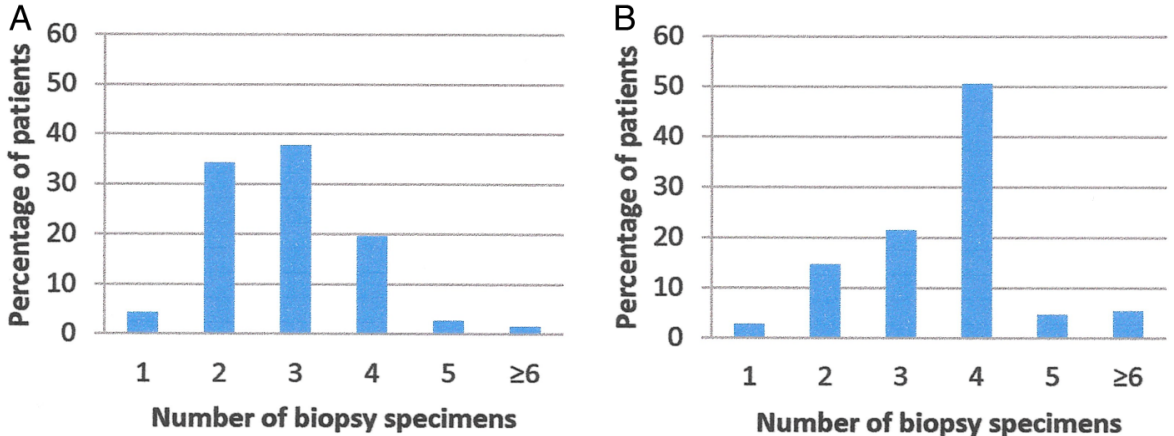
Table 2 Differences between audit and reaudit groups in terms of number of biopsy specimens submitted for histopathological analysis and diagnostic rate of coeliac disease

\begin{tabular}{|c|c|c|c|c|c|}
\hline & \multicolumn{2}{|c|}{ Audit (Aug 2014-May 2015) } & \multicolumn{2}{|c|}{$\begin{array}{l}\text { Reaudit (Feb 2016-May } \\
\text { 2016) }\end{array}$} & \multirow{2}{*}{ p Value } \\
\hline & Number & Per cent & Number & Per cent & \\
\hline \multicolumn{3}{|c|}{ Number of biopsy specimens } & & & $<0.001$ \\
\hline$<4$ & 705 & 76.3 & 109 & 39.2 & \\
\hline$\geq 4$ & 219 & 23.7 & 169 & 60.8 & \\
\hline Mean & 2.86 & - & 3.56 & - & $<0.001$ \\
\hline Coeliac disease & 32 & 3.5 & 21 & 7.6 & 0.004 \\
\hline
\end{tabular}

patients with no known diagnosis of $\mathrm{CD}$, in whom this diagnosis is being considered at endoscopy. This remained suboptimal even when the clinical indication for biopsies was 'suspected coeliac disease'. Although compliance only reached $60.8 \%$ in the reaudit, we have been able to demonstrate that simple interventional measures to raise awareness among endoscopists can be successful in significantly improving compliance to guidance. Potential barriers to adherence, as previously suggested in the literature ${ }^{7}$ include lack of awareness among clinicians of these guidelines and the extra time required to take a larger number of samples due to multiple passes of the biopsy forceps.

Of the 18 endoscopists who performed the majority of endoscopies during our study periods, only nine were present at the Endoscopy User Group meeting (November 2015) where we presented the results of our audit and encouraged endoscopists to comply with guidelines. Furthermore, a number of clinicians performing endoscopies in our trust are nor regular trust clinicians, but rather are from external agencies. Thus, reasons for failing to reach a higher rate of compliance in our postintervention group may include the fact that the recommendations from our initial study were not taken up by all clinicians involved, and potentially our message did not get across to everyone. The next stage of our study involves a more targeted training approach, to also include endoscopy nurses who are in a position to request clinicians to take an adequate number of biopsies. A formal assessment of individual clinicians' perceptions through questionnaires may further clarify barriers to compliance, and potentially help us to improve it further.

The diagnostic rate of $\mathrm{CD}$ doubled following implementation of changes $(7.6 \%$ in the reaudit vs $3.5 \%$ in the audit). There were no statistically significant differences between the audit and reaudit groups in terms of baseline characteristics, hence why we postulate that the higher probability of diagnosing $\mathrm{CD}$ in the reaudit group is likely attributable to the higher proportion of patients having $\geq 4$ biopsies taken. In fact, within each group there was a significant increase in the probability of a CD diagnosis when patients with $<4$ biopsy specimens were compared with those with $\geq 4$ specimens. Lebowhl et $a l^{7}$ also reported a doubling of the diagnostic rate of $\mathrm{CD}$ when four or more specimens are submitted for histology.
This raises the question of whether new diagnoses of CD are being missed due to inadequate number of biopsies submitted. CD is believed to be largely underdiagnosed. $^{9}$ A number of reasons for this have been suggested in the literature including the patchy nature of mucosal changes in CD, yield of biopsy samples that are inadequate for histological analysis and variability in histopathology reporting. ${ }^{10-12}$ Our results and those of similar studies ${ }^{7} 8$ strongly favour the submission of an adequate number of duodenal biopsies for histopathological analysis when a diagnosis of CD is considered.

The GEE analysis confirmed that while adjusting for clustering by endoscopists, the odds of compliance to guidelines still significantly increased in our reaudit cohort. We found that a positive serology result and 'suspected coeliac disease' as the principal indication for biopsy to be independent predictors of the likelihood of adhering to guidelines. In their study with a larger sample size, Lebowhl et $a l^{7}$ also found increasing age to be associated with decreased odds of submitting for biopsy specimens.

\section{Limitations}

Our study was retrospective and performed using electronic records. With the lack of information regarding clinical CD diagnosis, we formulated definitions of CD based on histology and serology. We did not have complete data on gross endoscopic findings, which are inconsistently documented by endoscopists; these would be best collected in a prospective design. Our reaudit sample size is also relatively small, and our study limited to a single centre. We have not quantified the exact effect that our intervention has had on improving compliance. However, all other factors have remained constant across the two periods of study-see table 1therefore allowing us to conclude that our intervention at least partly explains the improvement. We do aim to carry out more focused training and qualitatively measure its effect on clinicians' practice before carrying out a further reaudit.

\section{CONCLUSION}

Despite these limitations, our results are in line with previous studies. Our study has reiterated the fact that 
adherence to BSG recommendations on the number of duodenal biopsies taken at endoscopy when CD is suspected does improve the diagnostic yield of CD. Simple measures to increase awareness of this issue can improve local compliance, with a potential improved detection of CD. We also highlight the fact that despite the existence of the BSG guidelines, overall compliance remains quite poor at our centre, which may affect the diagnosis of CD. Our results should prompt other centres to assess their practice.

Acknowledgements We thank audit department, Barnsley Hospital; Rachael Sanderson, Lead Nurse Endoscopy, Barnsley Hospital; Christopher Barton, Clinical Effectiveness Assistant, Barnsley Hospital.

Contributors NH was involved in conception and design; acquisition, analysis and interpretation of the data; drafting of the article; critical revision of the article for important intellectual content; final approval of the article; and agreed to be accountable for all aspects of the work in ensuring that questions related to the accuracy or integrity of any part of the work are appropriately investigated and resolved. WA was involved in conception and design; acquisition of data; critical revision of the article for important intellectual content; final approval of the article; and agreed to be accountable for all aspects of the work in ensuring that questions related to the accuracy or integrity of any part of the work are appropriately investigated and resolved. MHS was involved in conception and design; critical revision of the article for important intellectual content; final approval of the article; and agreed to be accountable for all aspects of the work in ensuring that questions related to the accuracy or integrity of any part of the work are appropriately investigated and resolved. MHS is responsible for overall content as guarantor.

Competing interests None declared.

Provenance and peer review Not commissioned; externally peer reviewed.

Data sharing statement No additional data are available.
Open Access This is an Open Access article distributed in accordance with the Creative Commons Attribution Non Commercial (CC BY-NC 4.0) license, which permits others to distribute, remix, adapt, build upon this work noncommercially, and license their derivative works on different terms, provided the original work is properly cited and the use is non-commercial. See: http:// creativecommons.org/licenses/by-nc/4.0/

\section{REFERENCES}

1. Mustalahti K, Catassi $\mathrm{C}$, Reunanen A, et al. The prevalence of coeliac disease in Europe: results of a centralized, internationa mass screening project. Ann Med 2010;42:587-95.

2. Sanders DS, Carter MJ, Hurlstone DP, et al. Association of adult coeliac disease with irritable bowel syndrome: a case-control study in patients fulfilling ROME II criteria referred to secondary care. Lancet 2001;358:1504-8.

3. Ludvigsson JF, Leffler DA, Bai JC, et al. The Oslo definitions for coeliac disease and related terms. Gut 2013;62:43-52.

4. Catassi C, Bearzi I, Holmes GK. Association of celiac disease and intestinal lymphomas and other cancers. Gastroenterology 2005;128:S79-86.

5. Freeman HJ. Neoplastic disorders in 100 patients with adult celiac disease. Can J Gastroenterol 1996;10:163-6.

6. Ludvigsson JF, Bai JC, Biagi F, et al. Diagnosis and management of adult coeliac disease: guidelines from the British Society of Gastroenterology. Gut 2014;63:1210-28.

7. Lebwohl B, Kapel RC, Neugut Al, et al. Adherence to biopsy guidelines increases coeliac disease diagnosis. Gastrointest Endosc 2011;74:103-9.

8. Pais WP, Duerksen DR, Pettigrew NM, et al. How many duodenal biopsy specimens are required to make a diagnosis of celiac disease? Gastrointest Endosc 2008;67:1082-7.

9. Green $\mathrm{PH}$. Where are all those patients with coeliac disease? Am J Gastroenterol 2007;102:1461-3.

10. Latorre M, Lagana SM, Freedberg DE, et al. Endoscopic biopsy technique in the diagnosis of celiac disease: one bite or two? Gastrointest Endosc 2015;81:1228-33.

11. Gonzalez S, Gupta A, Cheng J, et al. Prospective study of the role of duodenal bulb biopsies in the diagnosis of celiac disease. Gastrointest Endosc 2010;72:758-65.

12. Arguelles-Grande C, Tennyson CA, Lewis SK, et al. Variability in small bowel histopathology reporting between different pathology practice settings: impact on the diagnosis of coeliac disease. $J$ Clin Pathol 2012;65:242-7. 